\title{
The origin and underlying driving forces of the SARS-CoV-2 outbreak
}

\author{
Shu-Miaw Chaw ${ }^{1 \dagger}$, Jui-Hung Tai ${ }^{1,2}$, Shi-Lun Chen ${ }^{3}$, Chia-Hung Hsieh ${ }^{4}$, Sui-Yuan Chang ${ }^{5}$, Shiou-Hwei Yeh ${ }^{6}$,
} Wei-Shiung Yang ${ }^{2}$, Pei-Jer Chen ${ }^{2}$ and Hurng-Yi Wang ${ }^{2,7^{*+}}$ (D)

\begin{abstract}
Background: SARS-CoV-2 began spreading in December 2019 and has since become a pandemic that has impacted many aspects of human society. Several issues concerning the origin, time of introduction to humans, evolutionary patterns, and underlying force driving the SARS-CoV-2 outbreak remain unclear.
\end{abstract}

Method: Genetic variation in 137 SARS-CoV-2 genomes and related coronaviruses as of 2/23/2020 was analyzed.

Result: After correcting for mutational bias, the excess of low frequency mutations on both synonymous and nonsynonymous sites was revealed which is consistent with the recent outbreak of the virus. In contrast to adaptive evolution previously reported for SARS-CoV during its brief epidemic in 2003, our analysis of SARS-CoV-2 genomes shows signs of relaxation. The sequence similarity in the spike receptor binding domain between SARS$\mathrm{CoV}-2$ and a sequence from pangolin is probably due to an ancient intergenomic introgression that occurred approximately 40 years ago. The current outbreak of SARS-CoV-2 was estimated to have originated on 12/11/2019 (95\% HPD 11/13/2019-12/23/2019). The effective population size of the virus showed an approximately 20-fold increase from the onset of the outbreak to the lockdown of Wuhan (1/23/2020) and ceased to increase afterwards, demonstrating the effectiveness of social distancing in preventing its spread. Two mutations, 845 in orf8 protein and $251 \mathrm{~V}$ in orf3 protein, occurred coincidentally with human intervention. The former first appeared on 1/5/2020 and plateaued around 1/23/2020. The latter rapidly increased in frequency after $1 / 23 / 2020$. Thus, the roles of these mutations on infectivity need to be elucidated. Genetic diversity of SARS-CoV-2 collected from China is two times higher than those derived from the rest of the world. A network analysis found that haplotypes collected from Wuhan were interior and had more mutational connections, both of which are consistent with the observation that the SARS-CoV-2 outbreak originated in China.

Conclusion: SARS-CoV-2 might have cryptically circulated within humans for years before being discovered. Data from the early outbreak and hospital archives are needed to trace its evolutionary path and determine the critical steps required for effective spreading.

Keywords: Positive selection, Population genetics, Coronavirus, Mutational bias

\footnotetext{
* Correspondence: hurngyi@ntu.edu.tw

†Shu-Miaw Chaw and Hurng-Yi Wang contributed equally to this work.

${ }^{2}$ Graduate Institute of Clinical Medicine, College of Medicine, National Taiwan

University, Taipei, Taiwan

${ }^{7}$ Institute of Ecology and Evolutionary Biology, National Taiwan University,

Taipei, Taiwan

Full list of author information is available at the end of the article
} 


\section{Background}

A newly emerging coronavirus was detected in patients during an outbreak of respiratory illnesses starting in mid-December of 2019 in Wuhan, the capital of Hubei Province, China [1-3]. Due to the similarity of its symptoms to those induced by the severe acute respiratory syndrome (SARS) and genome organization similarity, the causal virus was named SARS-CoV-2 by the International Committee on Taxonomy of Viruses [4]. As of 3/16/2020, 167,515 cases of SARS-CoV-2 infection have been confirmed in 114 countries, causing 6606 fatalities. As a result, WHO declared the first pandemic caused by a coronavirus on 3/11/2020 (https://www.who.int/emergencies/diseases/novel-coronavirus-2019/situation-re-

ports). As the virus continues to spread, numerous strains have been isolated and sequenced. On 3/18/2020, more than 500 complete or nearly complete genomes have been sequenced and made publicly available.

SARS-CoV-2 is the seventh coronavirus found to infect humans. Among the other six, SARS-CoV and MERS$\mathrm{CoV}$ can cause severe respiratory illness, whereas 229E, HKU1, NL63, and OC43 produce mild symptoms [5]. Current evidence strongly suggests that all human associated coronaviruses originated from other animals, such as bats and rodents [5, 6]. While SARS-CoV-2 shares similar genomic structure with other coronaviruses [7-10], its sequence differs substantially from some of the betacoronaviruses that infect humans, such as SARS-CoV (approximately $76 \%$ identity), MERS-CoV (43\% identity), and HKU-1 (33\% identity), but exhibits 96\% similarity to a coronavirus collected in Yunnan Province, China from a bat, Rhinolophus affinis. Therefore, SARS-CoV-2 most likely originated from bats $[2,11]$.

Several issues concerning the origin, time of virus introduction to humans, evolutionary patterns, and the underlying driving force of the SARS-CoV-2 outbreak remain to be clarified $[12,13]$. Here, we analyzed genetic variation of SARS-CoV-2 and its related coronaviruses. We discuss how mutational bias influences genetic diversity of the virus and attempt to infer forces that shape SARS-CoV-2 evolution.

\section{Methods}

\section{Data collection}

137 complete SARS-CoV-2 genomes were downloaded from the Global Initiative on Sharing Avian Influenza Data (GISAID, https://www.gisaid.org/) (Supplementary Table 1). Related coronavirus sequences, including those from five related bat sequences (RaTG13, HUK3-1, ZC45, ZXC-21, and GX2013), two pangolins (each from Guangdong (pangolin_2019) and Guangxi (pangolin_ 2017)), were downloaded from GenBank (https:// www.ncbi.nlm.nih.gov/nucleotide/). Nucleotide positions and coding sequences (CDSs) of SARS-CoV-2 were anchored to the reference genome NC_045512. CDS annotations of other coronaviruses were downloaded from GenBank.

\section{Sequence analyses and phylogeny construction}

CDSs were aligned based on translated amino acid sequences using MUSCLE v3.8.31 [14], and backtranslated to their corresponding DNA sequences using TRANALIGN software from the EMBOSS package (http://emboss.open-bio.org/) [15]. Nucleotide diversity, including number of segregating sites, Watterson's estimator of $\theta[16]$, and nucleotide diversity $(\pi)$ [17], was estimated using MEGA-X [18]. MEGA-X was also used for phylogenetic construction. Phylogenetic relationships were constructed using the neighbor-joining method based on Kimura's two-parameter model implemented in MEGA-X. Number of nonsynonymous changes per nonsynonymous site $(\mathrm{dN})$ and synonymous changes per synonymous site (dS) among genomes were estimated based Li-Wu-Luo's method [19] implemented in MEGA-X and PAML 4 [20]. The RDP file for the haplotype network analyses was generated using DnaSP 6.0 [21] and input into Network 10 (https://www.fluxusengineering.com/) to construct the haplotype network using the median joining algorithm. Four haplotype test implemented in DnaSp was applied to test for possible recombination event.

The mutation rate of SARS-CoV-2 and the time to the most recent common ancestor (TMRCA) of virus isolates were estimated by an established Bayesian MCMC approach implemented in BEAST version 1.10.4 [22]. The sampling dates were incorporated into TMRCA estimation. The analysis was performed using the HKY model of nucleotide substitution assuming an uncorrelated lognormal molecular clock [23]. We linked substitution rates for the first and second codon positions and allowed independent rates in the third codon position. We performed two independent runs with $3 \times 10^{8}$ MCMC steps and the results were combined. Log files were checked using Tracer (http://beast.bgio.ed.ac.uk/ Tracer). Effective sample sizes were $>300$ for all parameters.

\section{Results}

Molecular evolution of SARS-COV-2 and related coronaviruses

The resulting phylogeny reveals that RaTG13 is the closest relative of SARS-COV-2, followed by pangolin_2019 and pangolin_2017, then CoVZC45 and CoVZXC21, and other SARS-related sequences as outgroups (Supplementary Fig. 1). According to general time reversible model, transition occurred more frequent than transversion with C-T and A-G changes account for 45 and $28 \%$, respectively, of all six types of nucleotide changes. We 
next estimated the strength of selection for each coding region using the $\mathrm{dN}$ and $\mathrm{dS}$. While purifying selection tends to remove amino acid-altering mutations, thus reducing $\mathrm{dN}$ and $\mathrm{dN} / \mathrm{dS}$, positive selection has the opposite effect, increasing $\mathrm{dN}$ and $\mathrm{dN} / \mathrm{dS}$ [24]. Between SARSCoV-2 and RaTG13, orf8 gene exhibits the highest $\mathrm{dN}$ (0.032 highlighted in bold in Table 1$)$, followed by spike (0.013) and orf7 (0.011), all above the genome average of 0.007 (Table 1 ). $\mathrm{dS}$ varies greatly among CDSs with the highest of 0.313 in spike and the lowest of 0.018 in envelope (genome average 0.168 ). Finally, $\mathrm{dN} / \mathrm{dS}$ is the highest in orf8 (0.105), followed by orf7 (0.061) and orf3 (0.060), with the genome average of 0.042. Since spike shows both high $\mathrm{dS}$ and $\mathrm{dN}$, its protein evolution rate $(\mathrm{dN} / \mathrm{dS})$ is only 0.040 . Thus, while the coronavirus evolved very rapidly, it has actually been under tremendous selective constraint [13].

Spike protein similarity between SARS-CoV-2 and pangolin_2019 led to the idea that the receptor binding domain (RBD) within the SARS-CoV-2 spike protein originated from pangolin_2019 via recombination [2528 ]. If that were the case, we would expect the divergence at synonymous sites $(\mathrm{dS})$ to also be reduced in the $\mathrm{RBD}$ region. However, while $\mathrm{dN}$ in the $\mathrm{RBD}$ region is 0.023 , approximately one third of the estimate for the rest of the spike gene (0.068), dS in the RBD (0.710) is actually slightly higher than in the rest of the spike sequence (0.651). This argues against the recombination scenario. We noticed that the dS of the whole spike and the RBD, are 2- and 3-fold, respectively, higher than the genome average. Since synonymous sites are typically less influenced by selection, the increased divergence in dS may require further investigation.

\section{Genetic variation of SARS-CoV-2}

We downloaded 137 SARS-CoV-2 genomes available from GISAID as of $2 / 23 / 2019$. The coding regions were aligned and 223 mutations were identified with 68 synonymous and 155 nonsynonymous changes. The directionality of changes was inferred based on the RaTG13

Table 1 Pairwise comparison of nonsynonymous ( $\mathrm{dN}$; above slash) and synonymous (dS; below slash) divergence between SARSCoV-2, RaTG13, and Pangolin_2019 of different coding regions

\begin{tabular}{|c|c|c|c|c|}
\hline Gene & Length (aa) & SARS-CoV-2 vs RaTG13 & SARS-CoV-2 vs Pangolin_2019 & RaTG13 vs Pangolin_2019 \\
\hline \multirow[t]{2}{*}{ All } & 9555 & $0.007 / 0.168$ & $0.024 / 0.469$ & $0.025 / 0.467$ \\
\hline & & $(0.042)$ & $(0.051)$ & $(0.054)$ \\
\hline \multirow[t]{2}{*}{ orf1a } & 4330 & $0.008 / 0.166$ & $0.024 / 0.472$ & $0.023 / 0.472$ \\
\hline & & $(0.048)$ & $(0.051)$ & $(0.049)$ \\
\hline \multirow[t]{2}{*}{ orf1b } & 2692 & $0.003 / 0.126$ & $0.008 / 0.505$ & $0.010 / 0.515$ \\
\hline & & $(0.024)$ & $(0.016)$ & $(0.019)$ \\
\hline \multirow[t]{2}{*}{ spike } & 1219 & $0.013 / 0.313$ & $0.068 / 0.651$ & $0.073 / 0.680$ \\
\hline & & $(0.040)$ & $(0.104)$ & $(0.107)$ \\
\hline \multirow[t]{2}{*}{ RBD of spike ${ }^{A}$} & 219 & $0.055 / 0.511$ & $0.023 / 0.710$ & $0.058 / 0.863$ \\
\hline & & $(0.107)$ & $(0.032)$ & $(0.068)$ \\
\hline \multirow[t]{2}{*}{ orf3 } & 274 & $0.009 / 0.156$ & $0.019 / 0.285$ & $0.019 / 0.261$ \\
\hline & & $(0.060)$ & $(0.066)$ & $(0.072)$ \\
\hline \multirow[t]{2}{*}{ envelope } & 75 & 0/0.018 & 0/0.037 & 0/0.018 \\
\hline & & (0) & (0) & (0) \\
\hline \multirow[t]{2}{*}{ matrix } & 221 & $0.004 / 0.186$ & $0.010 / 0.299$ & $0.006 / 0.317$ \\
\hline & & $(0.021)$ & $(0.033)$ & $(0.019)$ \\
\hline \multirow[t]{2}{*}{ orf6 } & 60 & 0/0.099 & $0.014 / 0.220$ & $0.014 / 0.345$ \\
\hline & & (0) & $(0.062)$ & $(0.040)$ \\
\hline \multirow[t]{2}{*}{ orf7 } & 121 & $0.011 / 0.177$ & $0.018 / 0.275$ & $0.029 / 0.329$ \\
\hline & & $(0.061)$ & $(0.066)$ & $(0.088)$ \\
\hline \multirow[t]{2}{*}{ orf8 } & 121 & $0.032 / 0.303$ & $0.025 / 0.362$ & $0.017 / 0.391$ \\
\hline & & $(0.105)$ & (0.069) & $(0.042)$ \\
\hline \multirow[t]{2}{*}{ nucleocapsid } & 415 & $0.005 / 0.124$ & $0.011 / 0.145$ & $0.010 / 0.125$ \\
\hline & & $(0.042)$ & $(0.076)$ & $(0.080)$ \\
\hline
\end{tabular}




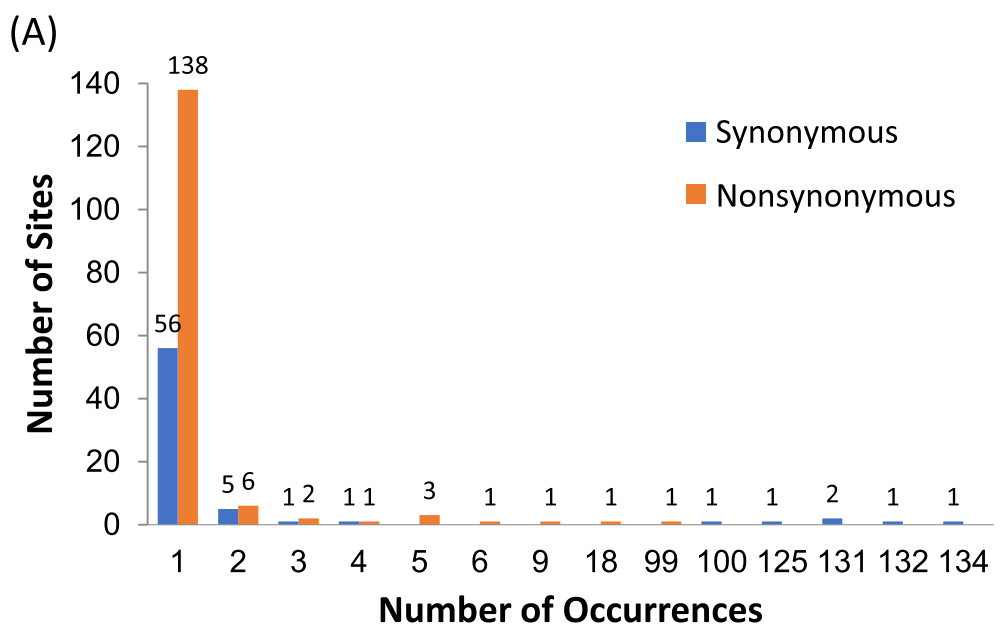

(B)

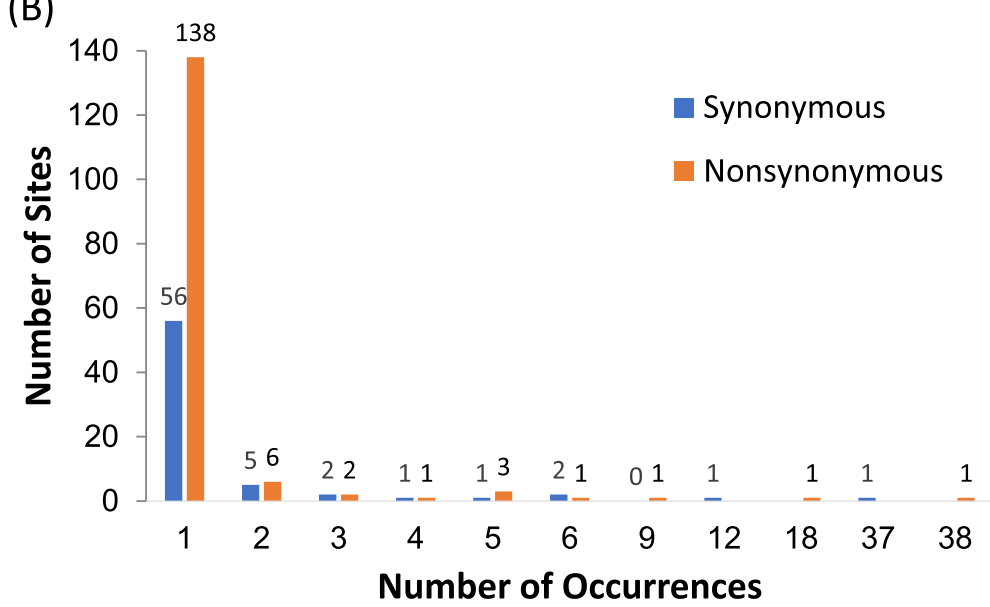

Fig. 1 Frequency spectra of SARS-CoV-2. The mutation frequency in 137 SARS-CoV-2 genomes is depicted on the $x$ axis, and the $y$ axis shows the number of sites in which mutations occurred. a The derived nucleotides were inferred by referencing SARS-CoV-2 genomes to the RaTG13 genome. $\mathbf{b}$ The direction of changes was cross-referenced with the haplotype network in Fig. 2

sequence. Frequency spectra of both synonymous and nonsynonymous changes are skewed. While the former shows excess of both high and low frequency mutations, the latter mainly exhibits an excess of low frequency changes (Fig. 1a). The excess of low frequency mutations is consistent with the recent origin of SARS-CoV-2 [29]. Both population reduction and positive selection can increase high frequency mutations $[30,31]$. However, the first scenario is contradicted by the recent origin of the virus. If positive selection has been operating, we would expect an excess of high frequency non-synonymous as well as synonymous changes. Furthermore, the ratio of nonsynonymous to synonymous $(\mathrm{N} / \mathrm{S})$ changes is $2.46(138 / 56)$ among singleton variants, but only 1.23 (16/13) among non-singletons. Both the nonsynonymous frequency spectrum and N/S ratio demonstrate that the majority of amino acid-altering mutations did not reach to high frequency. Thus, evidence for positive selection is limited.
The skew of synonymous variants toward high frequency deserves further discussion, as it relates to the underlying force driving the SARS-CoV-2 outbreak. The puzzle is probably rooted in how high and low frequency mutations are inferred. The results shown in the Fig. 1a are based on an outgroup comparison. The divergence at synonymous sites between SARS-CoV-2 and RaTG13 is 17\%, approximately 3fold greater than between humans and rhesus macaques [32]. With such high level of divergence, the possibility of multiple substitutions cannot be ignored, especially since substitutions in coronavirus genomes are strongly biased toward transitions (see above). Indeed, among all nonsingleton mutations listed in Table $2,62 \%$ of the changes are $\mathrm{C}-\mathrm{T}$ transitions.

To get around the potential problem caused by multiple substitutions, we cross-referenced the course of changes using the SARS-CoV-2 haplotype network (Fig. 2) and phylogeny (Supplementary Fig. 2; Supplementary Table 2). 
Table 2 Non-singleton mutations detected across the sampled SARS-CoV-2 genomes

\begin{tabular}{|c|c|c|c|c|c|c|c|c|c|c|}
\hline \multirow{2}{*}{\multicolumn{2}{|c|}{$\begin{array}{l}\text { Genome position } \\
\text { Nonsynonymous }\end{array}$}} & \multirow[t]{2}{*}{ Gene } & \multirow[t]{2}{*}{ RaTG13 } & \multirow[t]{2}{*}{ Pangolin_2017 } & \multirow[t]{2}{*}{ Pangolin_2019 } & \multirow[t]{2}{*}{ Major allele } & \multirow[t]{2}{*}{ Minor allele } & \multicolumn{3}{|c|}{ amount of change } \\
\hline & & & & & & & & I & $\|$ & \\
\hline A & 614 & orf1ab & G & G & G & G & $A$ & 2 & & H116Q \\
\hline$B$ & 1190 & orf1ab & C & C & C & C & $\mathrm{T}$ & 3 & & P308S \\
\hline C & 5084 & orf1ab & A & A & A & A & G & 2 & & A1606T \\
\hline $\mathrm{D}$ & 9438 & orf1ab & C & C & C & C & $\mathrm{T}$ & 3 & & T3058l \\
\hline$E$ & 11,083 & orf1ab & G & $\mathrm{T}$ & G & G & $\mathrm{T}$ & 9 & & L3606F \\
\hline $\mathrm{F}$ & 18,488 & orf1ab & T & $\mathrm{T}$ & T & T & C & 2 & & I6074V \\
\hline G & 21,707 & $S$ & C & C & N/A & C & $\mathrm{T}$ & 5 & & $\mathrm{H} 48 \mathrm{Y}$ \\
\hline $\mathrm{H}$ & 22,661 & $S$ & G & G & G & G & $\mathrm{T}$ & 5 & & V366F \\
\hline । & 26,144 & orf3 & G & G & G & G & $\mathrm{T}$ & 18 & & G251V \\
\hline J & 27,147 & $M$ & G & G & G & G & C & 2 & & I208T \\
\hline K & 28,077 & orf8 & G & G & G & G & C & 4 & & V61L \\
\hline$L$ & 28,144 & orf8 & C & $C$ & C & T & C & 99 & 38 & L84S \\
\hline M & 28,854 & $N$ & C & C & $C$ & $C$ & $\mathrm{~T}$ & 5 & & S194L \\
\hline$N$ & 28,878 & $N$ & G & G & G & G & A & 6 & & S202N \\
\hline 0 & 29,019 & $N$ & A & A & A & A & $\mathrm{T}$ & 2 & & $\mathrm{D} 249 \mathrm{H}$ \\
\hline$P$ & 29,303 & $N$ & $C$ & $C$ & $C$ & $C$ & $\mathrm{~T}$ & 2 & & K343I \\
\hline \multicolumn{11}{|c|}{ Synonymous } \\
\hline$a$ & 2662 & orfiab & $C$ & $\mathrm{~T}$ & T & C & T & 3 & & C2397T \\
\hline$\beta$ & 8782 & orfiab & T & $\mathrm{T}$ & T & $C$ & $\mathrm{~T}$ & 100 & 37 & C8517T \\
\hline Y & 10,138 & orfiab & $\mathrm{T}$ & T & T & $C$ & $\mathrm{~T}$ & 134 & 3 & C9873T \\
\hline \multirow[t]{2}{*}{$\delta$} & 15,324 & orf1ab & $C$ & $C$ & $C$ & C & $\mathrm{T}$ & 2 & & C15059T \\
\hline & 17,373 & orf1ab & T & C & $\mathrm{T}$ & $C$ & $\mathrm{~T}$ & 132 & 5 & C17108T \\
\hline$\zeta$ & 18,060 & orf1ab & T & T & A & C & $\mathrm{T}$ & 131 & 6 & C17795T \\
\hline$\eta$ & 18,603 & orf1ab & $\mathrm{T}$ & $\mathrm{T}$ & C & T & A & 2 & & T18338C \\
\hline$\theta$ & 23,569 & $S$ & A & C & A & T & C & 2 & & T2007C \\
\hline 1 & 23,605 & $S$ & N/A & N/A & $\mathrm{N} / \mathrm{A}$ & T & G & 2 & & T2043G \\
\hline K & 24,034 & $S$ & T & C & $C$ & C & $\mathrm{T}$ & 131 & 6 & C2472T \\
\hline$\lambda$ & 24,325 & $S$ & A & A & A & A & G & 2 & & A2763G \\
\hline$\mu$ & 26,729 & $M$ & $\mathrm{~T}$ & T & T & T & C & 4 & & T207C \\
\hline v & 29,095 & $N$ & $\mathrm{~T}$ & $\mathrm{~T}$ & $\mathrm{~T}$ & C & T & 125 & 12 & C822T \\
\hline
\end{tabular}

I: Number of changes was inferred by outgroup comparison only

II: Number of changes was cross-referenced with the haplotype network of SARS-CoV-2;only numbers different from method I were shown

E: envelope; M: matrix; N: nucleocapsid; S: spike

The two analyses yield very different pictures. For example, the highest frequency derived mutation in Table 2 is a C-T synonymous change at 10138 (marked $\gamma$ in Fig. 2 and Table 2). All three sequences from Singapore share the $\mathrm{T}$ nucleotide also found in the RaTG13 outgroup. Using the outgroup comparison, the $C$ found in the rest of the human SARS-CoV-2 sequences is a derived mutation. However, the $\mathrm{T}$ at this position is restricted to genomes collected from Singapore on $2 / 4$ and 2/6/2020 and not found in earlier samples. It is thus more sensible to infer that this $\mathrm{T}$ is a back mutation derived from $\mathrm{C}$ rather than an ancestral nucleotide. Another synonymous change at position 24,034 occurred twice (C24034T) on different genomic backgrounds (marked $\mathrm{k}$ in Fig. 2). Although the outgroup sequence at this position is $\mathrm{T}$, it is more likely that the $\mathrm{C}$ at this position is the ancestral nucleotide. We observed a number of such back or repeated mutations. An A-T nonsynonymous change at 29019 (D249H in nucleocapsid protein, marked $\mathrm{O}$ in Fig. 2) also occurred twice.

Repeated mutations may be caused by intergenomic recombination. Indeed, the result of four haplotype test suggested that at least two recombination events may have occurred between positions 8782 and 11,083 and 


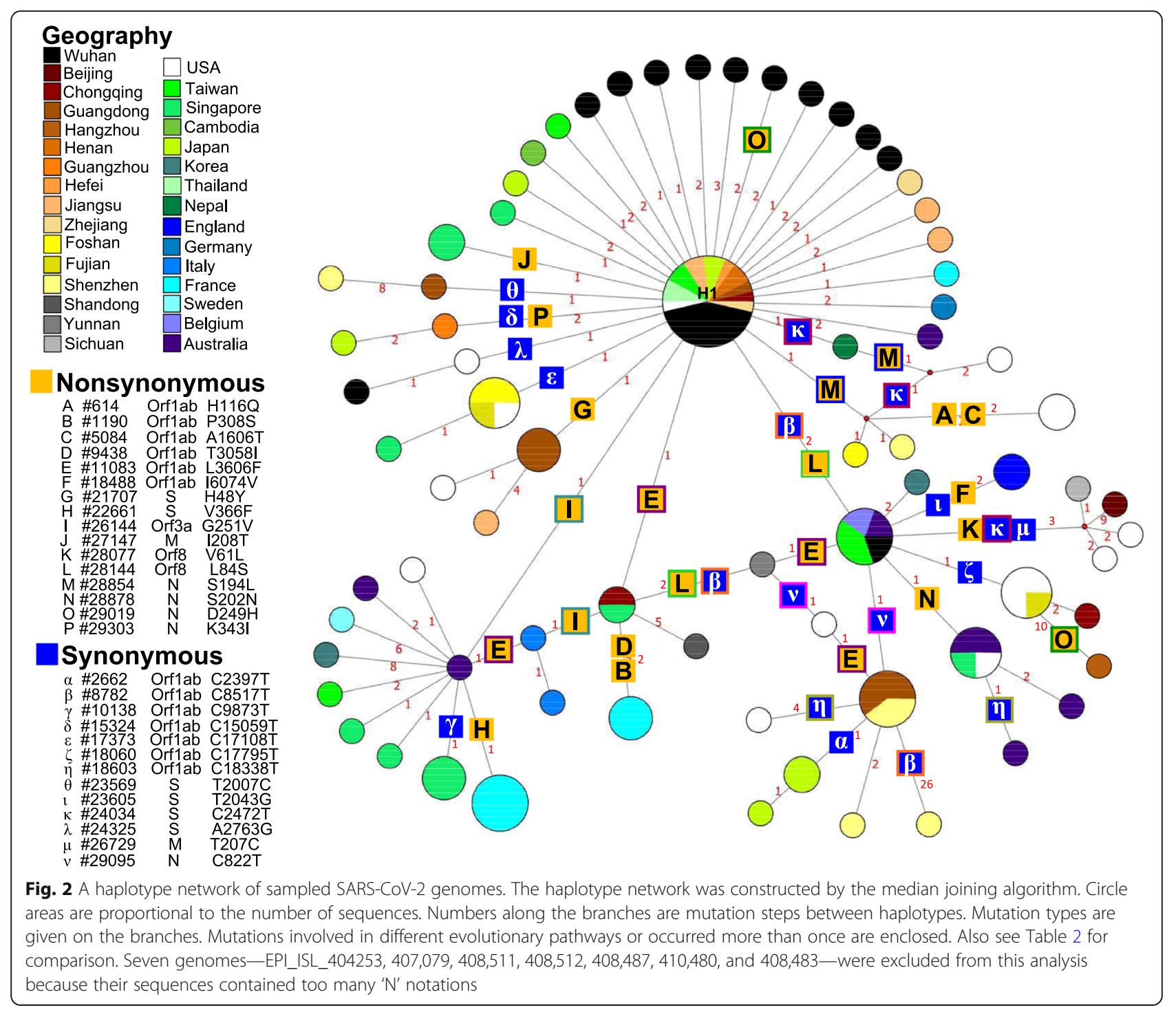

between 11,083 and 28,854 . We noticed that a sequence isolated on $1 / 21 / 2020$ from a patient in the United States (EPI_ISL_404253) exhibited Y (C or T) at both positions 8782 and 28,144 . Although, the possibility that two novel mutations might have occurred within this patient cannot be $100 \%$ ruled out, the alternative explanation that this patient may have been co-infected by two viral strains seems more plausible. After crossreferencing with the haplotype network and the phylogeny, all mutations listed as high frequency in Table 2 and Fig. 1a were re-assigned to the other side of the frequency spectra. We only see an excess of singleton mutations, consistent with a recent origin of SARS-CoV-2 (Fig. 1b) and suggesting that the virus has mainly evolved under constraint.

Perhaps the most controversial case is the T-C change at position 28,814 which alters Leucine $(\mathrm{L})$ to Serine $(\mathrm{S})$ in orf8 protein (L84S). Since both pangolin and RaTG13 have a $\mathrm{C}$ at this position (Table 2), Tang et al. suggested that $84 \mathrm{~L}$ is derived from $84 \mathrm{~S}$ in the human virus [13]. The $84 \mathrm{~S}$ was not discovered until $1 / 5 / 2020$, by which time 23 SARS-CoV-2 genomes have been sampled. After the first appearance, its frequency gradually increased, reaching approximately $30 \%$ by $1 / 23 / 2020$, suggesting that 84 S may exhibit some advantage over $84 \mathrm{~L}$. If genomes carrying $84 \mathrm{~S}$ were ancestral, it would be a challenge to explain its absence in early samplings. In addition, as mentioned above, $\mathrm{C}$ - $\mathrm{T}$ transitions are dominant in coronavirus evolution and multiple hits were observed in SARS-CoV-2 (Fig. 2 ). It is therefore possible that $28814 \mathrm{C}$ mutated to $\mathrm{T}$ after ancestral SARS-CoV-2 diverged from the common ancestor with RaTG13 and recently changed back to C. Finally, if $84 \mathrm{~L}$ is indeed a derived haplotype and has rapidly increased in its frequency by positive selection, we would expect haplotypes carrying $84 \mathrm{~L}$ to have accumulated more derived mutations than haplotypes with $84 S$. 
However, after correcting for mutational direction, the two haplotypes exhibited similar mutation frequency spectra (Supplementary Fig. 3). The alternative hypothesis that $84 \mathrm{~S}$ is a back mutation from $84 \mathrm{~L}$ is more plausible.

\section{Selection pressure on SARS-CoV-2}

In addition to L84S, a G-T transversion at 26144 which caused an amino acid change in orf3 protein (G251V) is also at intermediate frequency (Table 2). $251 \mathrm{~V}$ was first seen on $1 / 22 / 2020$ and gradually increased its frequency to $13 \%$ by our sampling date (Fig. 3 ). We note that the emergence of $84 \mathrm{~S}$ in orf8 and $251 \mathrm{~V}$ in orf3 proteins are consistent with the lockdown of Wuhan on 1/23/2020. The former first appeared in early January, gradually increased its frequency, and reached a plateau around $1 /$ $23 / 2020$. The latter showed up on $1 / 22 / 2020$ and rapidly increased its frequency within 2 weeks.

Based on Fig. 3, we divided the sampling course into two epidemic episodes, from the first sampled sequence $(12 / 24 / 2019)$ to before the lockdown of Wuhan $(1 / 21 / 2020)$ and from $1 / 22 / 2020$ to the date of the last sequence sampling $(2 / 23 / 2020)$. The $d N / d S$ of coding regions within the two episodes were estimated. As roughly $87 \%$ of mutations were singletons, many of these are probably sequencing errors, affecting synonymous and nonsynonymous sites equally and inflating our $\mathrm{dN} / \mathrm{dS}$ estimates. In addition, since $\mathrm{dN} / \mathrm{dS}$ is already extremely small in SARS-CoV-2 (Table 1), such inflation would have a large effect on
$\mathrm{dN} / \mathrm{dS}$ estimates. We therefore estimated $\mathrm{dN}$ and $\mathrm{dS}$ with (Supplementary Table 3) and without singletons (Table 3).

The $\mathrm{dN} / \mathrm{dS}$ of orf8 gene in episode I and II and orf3 gene in episode II show strong signatures of positive selection, consistent with increase of $84 \mathrm{~S}$ and $251 \mathrm{~V}$ frequency during these periods, and may suggest a role of adaptation (Table 3). The overall $\mathrm{dN} / \mathrm{dS}$ within each episode was 5-10 times higher than $\mathrm{dN} / \mathrm{dS}$ between coronavirus genomes derived from different species (Table 1). The elevated dN/dS of SARS-CoV-2 is either due to its adaptation to human hosts or relaxation of selection. For a recently emerged virus, it is reasonable to expect operation of positive selection at the early stage. In that case, the $\mathrm{dN} / \mathrm{dS}$ during episode I should be greater than during episode II [33, 34].

When singletons were included, $\mathrm{dN} / \mathrm{dS}$ in episode I was approximately $20 \%$ higher than that in episode II across the genome (Supplementary Table 3). In contrast, we observed the opposite result after removing singletons-i.e., $\mathrm{dN} / \mathrm{dS}$ in episode I was approximately $50 \%$ lower than that in episode II (Table 3). Therefore, the elevation of $\mathrm{dN} / \mathrm{dS}$ was most probably due to a relaxation in selection. We further divided episode I into Ia and $\mathrm{Ib}$, according to the appearance of $84 \mathrm{~S}$ in orf8 protein on $1 / 6 / 2020$. The genome-wide $\mathrm{dN} / \mathrm{dS}$ values were 0.27 and 0.23 for episode $1 \mathrm{a}$ and $1 \mathrm{~b}$, respectively (Supplementary Table 4). Therefore, as shown in the frequency spectra, the signature of positive selection is weak at the early stage of the epidemic.

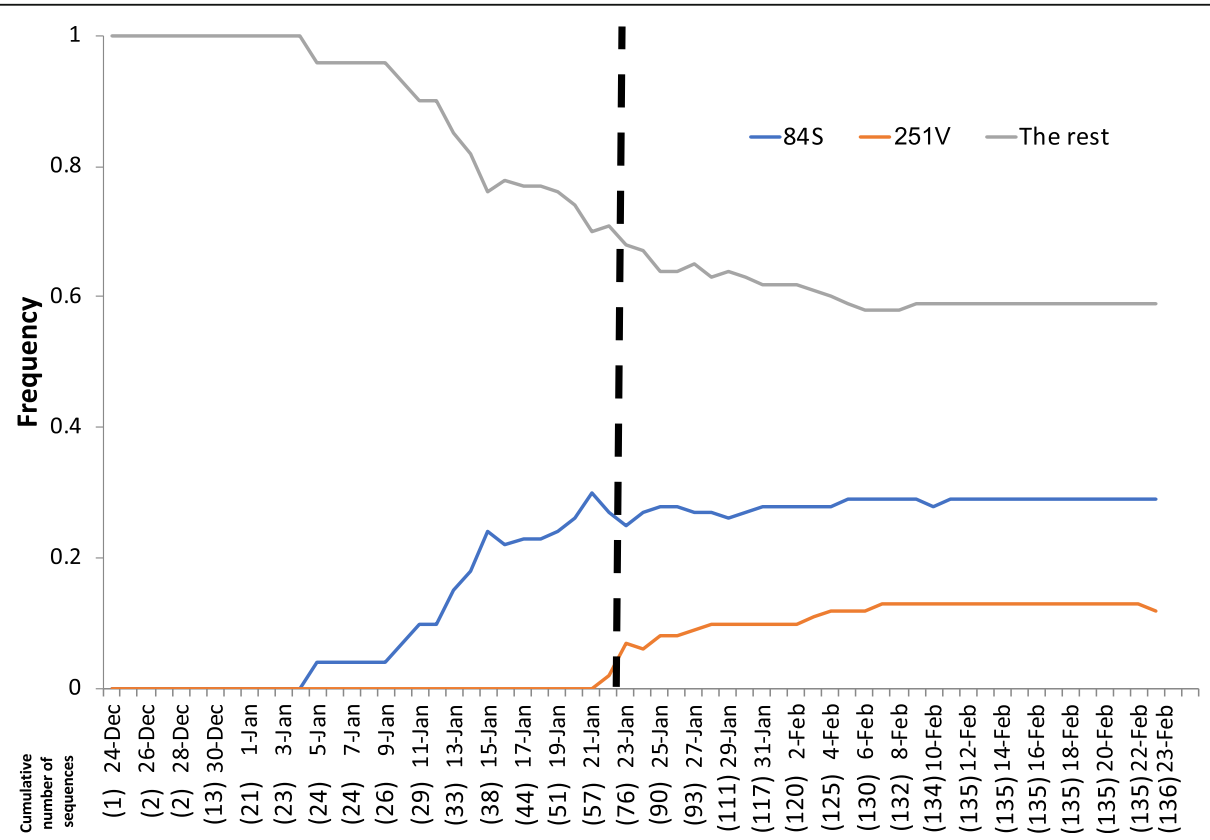

Fig. 3 Mutation frequency of $84 \mathrm{~S}$ in orf8 and $251 \mathrm{~V}$ in orf3 proteins. Numbers in parentheses are cumulative number of sequences on the indicated day. The dashed line indicates the date of the Wuhan lockdown 
Table 3 Comparison of $\mathrm{dN}, \mathrm{dS}$, and dN/dS estimates in the coding regions of SARS-CoV-2 without singleton between two episodes

\begin{tabular}{|c|c|c|c|c|c|c|}
\hline \multirow[t]{2}{*}{ Gene } & \multicolumn{2}{|c|}{ Episode I $(N=57)(2019 / 12 / 24-2020 / 1 / 21)$} & \multicolumn{2}{|c|}{ Episode II $(N=79)(2020 / 1 / 22-2020 / 2 / 23)$} & \multicolumn{2}{|c|}{ Episode I + II (2019/12/24-2020/2/23) } \\
\hline & $\mathrm{dN} \times 10^{4}$ & $\mathrm{dS} \times 10^{4}$ & $\mathrm{dN} \times 10^{4}$ & $\mathrm{dS} \times 10^{4}$ & $\mathrm{dN} \times 10^{4}$ & $d S \times 10^{4}$ \\
\hline & $\mathrm{dN} / \mathrm{dS}$ & & $\mathrm{dN} / \mathrm{dS}$ & & $\mathrm{dN} / \mathrm{dS}$ & \\
\hline \multirow[t]{2}{*}{ All } & 0.34 & 1.70 & 0.78 & 1.98 & 0.61 & 1.87 \\
\hline & 0.20 & & 0.39 & & 0.32 & \\
\hline \multirow[t]{2}{*}{ orf1a } & 0.10 & 1.46 & 0.37 & 2.15 & 0.26 & 1.85 \\
\hline & 0.07 & & 0.17 & & 0.14 & \\
\hline \multirow[t]{2}{*}{ orfib } & 0.06 & 0.81 & 0.08 & 1.60 & 0.07 & 1.29 \\
\hline & 0.07 & & 0.05 & & 0.05 & \\
\hline \multirow[t]{2}{*}{ spike } & 0.23 & 2.49 & 0.64 & 1.82 & 0.48 & 2.10 \\
\hline & 0.09 & & 0.35 & & 0.23 & \\
\hline \multirow[t]{2}{*}{ orf3 } & 0.00 & 0.00 & 5.30 & $(1.98)^{*}$ & 3.42 & $(1.87)^{*}$ \\
\hline & 0.00 & & 2.68 & & 1.83 & \\
\hline \multirow[t]{2}{*}{ envelope } & 0.00 & 0.00 & 0.00 & 0.00 & 0.00 & 0.00 \\
\hline & 0.00 & & 0.00 & & 0.00 & \\
\hline \multirow[t]{2}{*}{ matrix } & 0.00 & 4.60 & 0.97 & 3.37 & 0.57 & 3.86 \\
\hline & 0.00 & & 0.29 & & 0.15 & \\
\hline \multirow[t]{2}{*}{ orf6 } & 0.00 & 0.00 & 0.00 & 0.00 & 0.00 & 0.00 \\
\hline & 0.00 & & 0.00 & & 0.00 & \\
\hline \multirow[t]{2}{*}{ orf7 } & 0.00 & 0.00 & 0.00 & 0.00 & 0.00 & 0.00 \\
\hline & 0.00 & & 0.00 & & 0.00 & \\
\hline \multirow[t]{2}{*}{ orf8 } & 16.26 & $(1.70)^{*}$ & 15.84 & $(1.98)^{*}$ & 15.90 & $(1.87)^{*}$ \\
\hline & 9.57 & & 8.02 & & 8.51 & \\
\hline \multirow[t]{2}{*}{ nucleocapsid } & 1.16 & 7.31 & 2.98 & 4.26 & 2.25 & 5.61 \\
\hline & 0.16 & & 0.70 & & 0.40 & \\
\hline
\end{tabular}

*No synonymous mutation in this region was observed. The genome-wide dS value was used here. As the sequence EPI_ISL_411929 from South Korea did not have sampling date, it was excluded from this analysis

\section{The origin of SARS-CoV-2}

The estimated mutation rate of SARS-CoV-2 is $2.4 \times$ $10^{-3} /$ site/year with $95 \%$ highest posterior density (HPD) of $1.5-3.3 \times 10^{-3} / \mathrm{site} /$ year. The mutation rate at the third codon position is $2.9 \times 10^{-3} /$ site/year $(95 \% \mathrm{HPD}$ $1.8-4.0 \times 10^{-3} /$ site/year), which is in a good agreement with synonymous mutation rate of SARS-CoV, 1.67$4.67 \times 10^{-3} /$ site/year [34]. SARS-CoV-2 is estimated to have originated on 12/11/2019 (95\% HPD 11/13/2019$12 / 23 / 2019$ ). The initial effective population size of the virus was small, which is consistent with the recent origin of SARS-CoV-2. The population size showed approximately 20-fold increase from the onset of the outbreak to the lockdown of Wuhan $(1 / 23 / 2020)$ and ceased to increase afterwards, demonstrating the effectiveness of social distancing on preventing virus spread (Fig. 4). We have to point out that the TMRCA and epidemic growth curve estimation are strongly influenced by the sampling scheme. For example, since the earliest available genome was sampled on 12/24/2019 almost 1 month after the outbreak, the real origin of the current outbreak may actually be earlier than our estimation. In addition, all sequences from Wuhan were sampled before $01 / 05 / 2020$ which would have an influence on demographic estimation.

We estimated genetic variation, including the number of segregating sites, Watterson's estimator of $\theta$, and nucleotide diversity $(\pi)$ of the SARS-CoV-2. Since both $\pi$ and $\theta$ are estimators of $4 \mathrm{Nu}(\mathrm{N}$ and $\mathrm{u}$ are the effective population size and mutation rate, respectively), they should be close to each other at the mutation-drift equilibrium [35]. Because $\theta$ is strongly influenced by rare mutations which are common during recent population expansion [24], it is a better estimator of genetic diversity for SARS-CoV-2. For example, when all samples are considered, $\theta\left(13.92 \times 10^{-4}\right)$ is approximately eight times higher than $\pi\left(1.81 \times 10^{-4}\right.$, Table 4). Among samples collected from different locations, sequences from China 


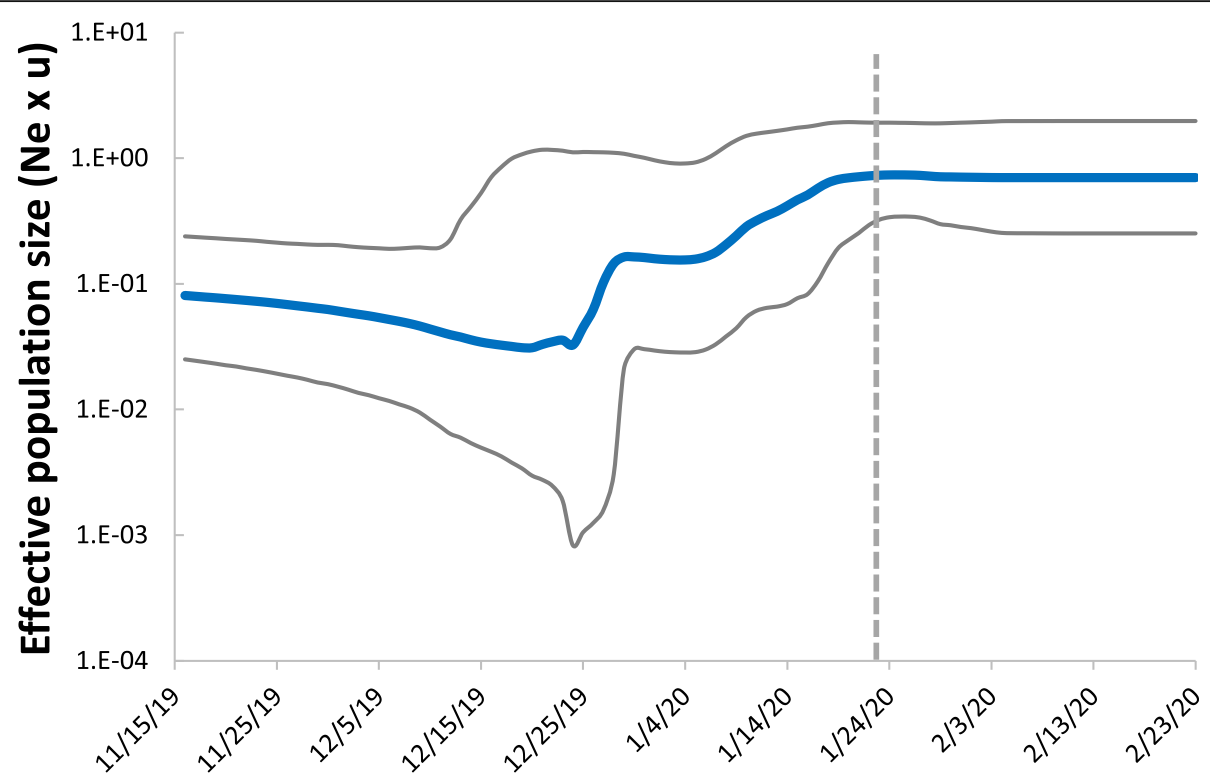

\section{Date}

Fig. 4 The epidemic growth curve for the SARS-CoV-2 outbreak. The three lines are the median (blue line) and 95\% HPD intervals (gray lines) of the Bayesian skyline plot $(m=5)$. Vertical dash line indicates the date of the Wuhan lockdown

exhibited higher genetic variation in terms of the number of segregating sites, $\theta$ and $\pi$, than the rest of the world combined, consistent with the observation that the outbreak originated in China, as the source populations are expected to exhibit higher genetic variation than derived populations [35].

The haplotype network also supports this notion (Fig. 2). Usually, ancestral haplotypes have a greater probability of being in the interior, have more mutational connections, and are geographically more widely distributed. The H1 haplotype is at the center of the network and is found in four countries and many places in China. In addition, a large portion of haplotypes is directly connected to H1. Therefore, it is likely that $\mathrm{H} 1$ is the ancestral haplotype. As $45 \%$ of $\mathrm{H} 1$ are found in Wuhan, this location is the most plausible origin of the ongoing pandemic.

\section{Discussion}

A close relationship between SARS-CoV-2 and pangolin_2019 at the amino acid level in the RBD region of the spike protein might be due to recent recombination $[25,26]$, data contamination, or convergent evolution. Since recent recombination and DNA contamination should affect synonymous and nonsynonymous sites equally, they can be convincingly rejected as great divergence at synonymous sites was observed in spite of similar amino acid sequences between the two genomes. While genotypic convergence may be observed in viruses repeatedly evolving under particular conditions, such as

Table 4 Estimated nucleotide diversity of SARS-CoV-2 across geographic regions

\begin{tabular}{lllll}
\hline Sample origin & Sample size & S & $\theta \times 10^{-4}$ & 13.92 \\
\hline Total & 137 & 223 & 11.38 & 1.81 \\
China & 64 & 157 & 3.76 & 2.10 \\
$\quad$ Wuhan & 24 & 41 & 9.59 & 1.16 \\
$\quad$ Rest of China & 40 & 119 & 5.71 & 2.62 \\
Rest of the World & 73 & 81 & 2.84 & 1.52 \\
$\quad$ USA & 17 & 62 & 4.63 & 1.71 \\
$\quad$ Rest of the World excluding USA & 56 & & \\
\hline
\end{tabular}

S: Number of segregating sites

$\theta$ : Nucleotide diversity based on Watterson [29]

$\pi$ : Nucleotide diversity based on Nei and Li [30] 
drug resistance and immune escape [36-39], it is otherwise rare. For adaptations that do not involve highly specialized conditions, divergent molecular pathways may develop and genotypic convergence would not be expected [40]. For example, SARS-CoV and SARS-CoV-2 both use the spike protein to bind human ACE2 [2], but five out of six critical amino acids within the RBD are different between these two viruses [27]. Since the SARS-CoV-2 and pangolin_2019 have diverged at about $47 \%$ of synonymous sites and infect different hosts, the idea that they share five out of six critical amino acids within RBD through convergent evolution seems far-fetched.

We therefore hypothesize that, instead of convergent evolution, the similarity of RBD between SARS-CoV-2 and pangolin_2019 was caused by an ancient intergenomic recombination. Assuming a synonymous substitution rate of $2.9 \times 10^{-3} /$ site/year, the recombination was estimated to have occurred approximately 40 years ago (95\% HPD: 31-69 years; divergence time $(\mathrm{t})=$ divergence $(\mathrm{dS}) /($ substitution rate $\times 2 \times 3$ ), considering $\mathrm{dS}$ in $\mathrm{RBD}$ is 3 -fold of genome average). The amino acids in the RBD region of the two genomes have been maintained by natural selection ever since, while synonymous substitutions have been accumulated. If this is true, SARS-CoV-2 may have circulated cryptically among humans for years before being recently noticed.

The ancient origin of SARS-CoV-2 is supported by its lack of a signature of adaptive evolution as shown by frequency spectra and dN/dS in samples from the recent epidemic. For a recently acquired virus, rapid evolution and a strong signature of positive selection are expected. For example, during its short epidemic in 2002-2003, several rounds of adaptive changes have been documented in SARS-CoV genomes [33, 34]. After adapting to its host, the virus may evolve under purifying or relaxed selection, exactly as we see in SARS-CoV-2. Therefore, it is important to sequence samples from the early outbreak and to examine hospital archives for the trace of SARS-CoV-2 ancestors. This information not only can help us to understand the evolutionary path of this virus but also unravel the critical steps for it to achieve effective spreading in humans.

In addition to the RBD, the SARS-CoV-2 spike protein also contains a small insertion of a polybasic cleavage site which was thought to be unique within the B lineage of betacoronaviruses [27]. However, a recent analysis of bats collected from Yunnan, China, identified a similar insertion in a sequence, RmYN02, closely related to SARS-CoV-2, providing strong evidence that such seemingly sorcerous site insertions can occur in nature [11]. Both the polybasic cleavage site in RmYN02 and RBD in pangolin_2019 suggest that, like with SARS-CoV [6], all genetic elements required to form SARS-CoV-2 may have existed in the environment. More importantly, they can be brought together by frequent intergenomic recombination (see Result). Nature never runs out of material to create new pathogens. It is not whether but when and where the next epidemic will occur.

There is a heated debate about the evolutionary forces influencing the trajectory of the L84S mutation in orf8 protein (http://virological.org/t/response-to-on-the-origin-and-continuing-evolution-of-sars-cov-2/418). While Tang et al. considered Serine is the ancestral amino acid [13], we present evidence that it is a back mutation. The majority of sequences in Wuhan were sampled before early January 2020 and most genomes carrying $84 S$ were found outside Wuhan after middle to late January 2020. The discrepancy in time and space impedes the effort to resolve the debate. It would require more sequences from the early stage of the epidemic to settle this issue. Regardless of its ancestral or derived status, we hypothesize that $84 \mathrm{~S}$ may confer some selective advantage. Unless the sampling scheme is deliberately skewed, it is difficult to explain such dramatic frequency gain of $84 \mathrm{~S}$, from 0 to $\sim 30 \%$ in 2 weeks. Oddly, its frequency ceased to increase after $1 / 23 / 2020$, when Wuhan was locked down. This coincidence prompts us to consider the effect of social distancing on virus transmission. Another line of evidence comes from the frequency increase of $215 \mathrm{~V}$ in orf3 protein. The $215 \mathrm{~V}$ first appeared on $1 / 22 / 2020$ and rapidly increased its frequency within 2 weeks.

Several studies suggested that the orf8 protein may function in viral replication, modulating endoplasmic reticulum stress, inducing apoptosis, and inhibiting interferon responses in host cells (41-45 [41-45]. During the SARS spread, frequency of several orf8 mutations fluctuated in accordance with different phases of the outbreak, suggesting that orf8 underwent adaptation during the SARS epidemic [34]. It is suggested that $84 \mathrm{~S}$ may induce structural disorder in the C-terminus of the protein and may generate a novel phosphorylation target for Serine/ Threonine kinases of the mammalian hosts [46].

SARS-CoV orf3 protein has been shown to activate NF- $K B$ and the NLRP3 inflammasome and causes necrotic cell death, lysosomal damage, and caspase-1 activation. In addition, orf3 is required for maximal SARS$\mathrm{CoV}$ replication and virulence. All of the above likely contributes to the clinical manifestations of SARS-CoV infection [47-49]. Therefore, these two mutations may have some functional consequences and be worth investigating further. By the time we prepared this manuscript, the $215 \mathrm{~V}$ frequency ceased to increase. However, a parallel mutation has occurred in a different genomic background, further supporting the idea that this mutation may require further study. 


\section{Conclusion}

In contrast to adaptive evolution previously reported for SARS-CoV in its brief epidemic, our analysis of SARSCoV-2 genomes shows signs of relaxation of selection which, in combination with an ancient intergenomic introgression in $\mathrm{RBD}$ of spike protein, suggests that SARS-CoV-2 might have cryptically circulated within humans for years before being recently noticed. Data from the early outbreak and hospital archives are needed to trace its evolutionary path and reveal critical steps required for effective spreading. We found that the lockdown of Wuhan is strongly associated with frequency fluctuations of $84 \mathrm{~S}$ in orf8 and $215 \mathrm{~V}$ in orf3 proteins and population size of the virus, suggesting the effectiveness of human intervention, such as social distancing, on preventing virus spread.

\section{Supplementary information}

Supplementary information accompanies this paper at https://doi.org/10 1186/s12929-020-00665-8.

Additional file 1: Supplementary Figure 1. The neighbor-joining tree of SARS-CoV-2 related coronaviruses constructed by concatenating coding sequences based on the Kimura 2-parameter model implemented in MEGA-X. Supplementary Figure 2. Unrooted neighbor-joining tree of SARS-CoV-2 constructed by concatenating coding sequences based on the Kimura 2-parameter model implemented in MEGA-X. Non-singleton changes are shown along the branches. The location of each sequence is given (above the slash) followed by its sampling date (below the slash). For multiple sequences sampled on the same date from the same location, the index, $a, b, c, d$, and etc. is given. Details are listed in Supplementary Table 2. Supplementary Figure 3. Frequency spectra of SARSCoV-2 carrying $84 \mathrm{~L}(n=98)(\mathrm{A})$ and $84 \mathrm{~S}(n=39)(\mathrm{B})$ in orf8 protein. The direction of changes was cross-referenced with the haplotype network shown in Fig. 2

Additional file 2: Supplementary Table 1. People contributed to sequence generation and sharing

Additional file 3: Supplementary Table 2.

Additional file 4: Supplementary Table 3. Comparison of $d \mathrm{~N}, \mathrm{dS}$, and $\mathrm{dN} / \mathrm{dS}$ in the coding regions of SARS-CoV-2 with singleton between different episodes. Supplementary Table 4. Comparison of dN, dS, and $\mathrm{dN} / \mathrm{dS}$ in the coding regions of SARS-CoV-2 without singleton between episode la and $\mathrm{lb}$.

\section{Acknowledgements}

The authors thank those who contributed to sequence generation and sharing (The detail is listed in SUPPLEMENTARY Table 1). We also thank Chung-I Wu, Wen-Ya Ko, and Yu-Ching Lan for their constructive comments and suggestions.

\section{Authors' contributions}

SMC co-conceptualized the study, obtained partial funding, and revised the manuscript. JHT and SLC analyzed the data. SYC and SHY guided the analyses. SMC, WSY, and PJC drafted and revised the manuscript. HYW designed the study, obtained funding, and wrote the manuscript.

\section{Funding}

This work was supported by Ministry of Science and Technology, National Taiwan University, and National Taiwan University, College of Medicine, Taipei, Taiwan to HYW (105-2628-B-002-015-MY3, 107-2321-B-002-004-, NTU109 L7806, NSC-131-5), and partially by a grant from Biodiversity Research Center, Academia Sinica to SMC.

\section{Availability of data and materials}

All genome sequences were downloaded from Global Initiative on Sharing Avian Influenza Data (GISAID, https://www.gisaid.org/) and GenBank (https:// www.ncbi.n/m.nih.gov/nucleotide/).

Ethics approval and consent to participate

Not applicable.

Consent for publication

Not applicable.

\section{Competing interests}

The authors declare that they have no competing interests.

\section{Author details}

${ }^{1}$ Biodiversity Research Center, Academia Sinica, Taipei, Taiwan. ${ }^{2}$ Graduate Institute of Clinical Medicine, College of Medicine, National Taiwan University, Taipei, Taiwan. ${ }^{3}$ Department of Life Science, National Taiwan Normal University, Taipei, Taiwan. ${ }^{4}$ Department of Forestry and Nature Conservation, Chinese Culture University, Taipei, Taiwan. ${ }^{5}$ Department of Clinical Laboratory Sciences and Medical Biotechnology, College of Medicine, National Taiwan University, Taipei, Taiwan. ${ }^{6}$ Department of Microbiology, College of Medicine, National Taiwan University, Taipei, Taiwan. ${ }^{7}$ Institute of Ecology and Evolutionary Biology, National Taiwan University, Taipei, Taiwan.

Received: 21 April 2020 Accepted: 28 May 2020

Published online: 07 June 2020

\section{References}

1. Ren LL, Wang YM, Wu ZQ, Xiang ZC, Guo L, Xu T, et al. Identification of a novel coronavirus causing severe pneumonia in human: a descriptive study Chin Med J. 2020

2. Zhou P, Yang XL, Wang XG, Hu B, Zhang L, Zhang W, et al. A pneumonia outbreak associated with a new coronavirus of probable bat origin. Nature. 2020;579(7798):270-3.

3. Wu F, Zhao S, Yu B, Chen YM, Wang W, Song ZG, et al. A new coronavirus associated with human respiratory disease in China. Nature. 2020;579(7798):265-9.

4. Gorbalenya A, Baker S, Baric R, de Groot R, Drosten C, Gulyaeva A, et al. The species Severe acute respiratory syndrome-related coronavirus: classifying 2019-nCoV and naming it SARS-CoV-2. Nat Microbiol. 2020;5:536-44.

5. Corman VM, Muth D, Niemeyer D, Drosten C. Hosts and sources of endemic human coronaviruses. Adv Virus Res. 2018;100:163-88.

6. Cui J, Li F, Shi ZL. Origin and evolution of pathogenic coronaviruses. Nat Rev Microbiol. 2019;17(3):181-92

7. Benvenuto D, Giovanetti M, Ciccozzi A, Spoto S, Angeletti S, Ciccozzi M. The 2019-new coronavirus epidemic: evidence for virus evolution. J Med Virol. 2020;92(4):455-9.

8. Wu A, Peng Y, Huang B, Ding X, Wang X, Niu P, et al. Genome composition and divergence of the novel coronavirus (2019-nCoV) originating in China. Cell Host Microbe. 2020;27(3):325-8.

9. Lu R, Zhao X, Li J, Niu P, Yang B, Wu H, et al. Genomic characterisation and epidemiology of 2019 novel coronavirus: implications for virus origins and receptor binding. Lancet. 2020;395(10224):565-74.

10. Chan JF, Kok KH, Zhu Z, Chu H, To KK, Yuan S, et al. Genomic characterization of the 2019 novel human-pathogenic coronavirus isolated from a patient with atypical pneumonia after visiting Wuhan. Emerg Microbes Infect. 2020;9(1):221-36

11. Zhou H, Chen X, Hu T, Li J, Song H, Liu Y, et al.. A novel bat coronavirus reveals natural insertions at the S1/S2 cleavage site of the Spike protein and a possible recombinant origin of HCoV-19. BioRxiv. 2020:2020.03.02.974139.

12. Wu C-I, Poo M-m. Moral imperative for the immediate release of 2019-nCoV sequence data. Natl Sci Rev 2020

13. Tang $X$, Wu C, Li X, Song $Y$, Yao X, Wu X, et al. On the origin and continuing evolution of SARS-CoV-2. Natl Sci Rev. 2020

14. Edgar RC. MUSCLE: multiple sequence alignment with high accuracy and high throughput. Nucleic Acids Res. 2004;32(5):1792-7.

15. Rice $\mathrm{P}$, Longden I, Bleasby A. EMBOSS: the European molecular biology open software suite. Trends Genet. 2000;16(6):276-7.

16. Watterson GA. On the number of segregating sites in genetical models without recombination. Theor Popul Biol. 1975;7(2):256-76. 
17. Nei M, Li WH. Mathematical model for studying genetic variation in terms of restriction endonucleases. Proc Natl Acad Sci U S A. 1979;76(10):5269-73.

18. Kumar S, Stecher G, Li M, Knyaz C, Tamura K. MEGA X: Molecular evolutionary genetics analysis across computing platforms. Mol Biol Evol. 2018;35(6):1547-9

19. Li WH, Wu Cl, Luo CC. A new method for estimating synonymous and nonsynonymous rates of nucleotide substitution considering the relative likelihood of nucleotide and codon changes. Mol Biol Evol. 1985;2(2):150-74.

20. Yang Z. PAML 4: phylogenetic analysis by maximum likelihood. Mol Biol Evol. 2007;24(8):1586-91.

21. Rozas J, Ferrer-Mata A, Sanchez-DelBarrio JC, Guirao-Rico S, Librado P, Ramos-Onsins SE, et al. DnaSP 6: DNA sequence polymorphism analysis of large data sets. Mol Biol Evol. 2017;34(12):3299-302.

22. Suchard MA, Lemey P, Baele G, Ayres DL, Drummond AJ, Rambaut A. Bayesian phylogenetic and phylodynamic data integration using BEAST 1. 10. Virus Evol. 2018;4(1).

23. Drummond AJ, Ho SY, Phillips MJ, Rambaut A. Relaxed phylogenetics and dating with confidence. PLoS Biol. 2006;4(5):e88.

24. Li W-H. Molecular evolution. Sunderland, Mass.: Sinauer Associates; 1997. XV, 487 p. p.

25. Wong MC, Javornik Cregeen SJ, Ajami NJ, Petrosino JF. Evidence of recombination in coronaviruses implicating pangolin origins of nCoV-2019. BioRxiv. 2020:2020.02.07.939207.

26. Xiao K, Zhai J, Feng Y, Zhou N, Zhang X, Zou J-J, et al.. Isolation and Characterization of 2019-nCoV-like Coronavirus from Malayan Pangolins. bioRxiv. 2020:2020.02.17.951335.

27. Andersen KG, Rambaut A, Lipkin WI, Holmes EC, Garry RF. The proximal origin of SARS-CoV-2. Nat Med. 2020,

28. Lam TT, Shum MH, Zhu HC, Tong YG, Ni XB, Liao YS, et al. Identifying SARSCoV-2 related coronaviruses in Malayan pangolins. Nature. 2020

29. Zhang C, Wang M. Origin time and epidemic dynamics of the 2019 novel coronavirus. bioRxiv. 2020:2020.01.25.919688

30. Fay JC, Wu Cl. Hitchhiking under positive Darwinian selection. Genetics. 2000;155(3):1405-13.

31. Zeng K, Shi S, Wu Cl. Compound tests for the detection of hitchhiking under positive selection. Mol Biol Evol. 2007;24(8):1898-908.

32. Wang HY, Chien HC, Osada N, Hashimoto K, Sugano S, Gojobori T, et al. Rate of evolution in brain-expressed genes in humans and other primates. PLoS Biol. 2007:5(2):e13.

33. Yeh SH, Wang HY, Tsai CY, Kao CL, Yang JY, Liu HW, et al. Characterization of severe acute respiratory syndrome coronavirus genomes in Taiwan: molecular epidemiology and genome evolution. Proc Natl Acad Sci U S A. 2004;101(8):2542-7.

34. Chinese SMEC. Molecular evolution of the SARS coronavirus during the course of the SARS epidemic in China. Science. 2004;303(5664):1666-9.

35. Hahn MW. Molecular population genetics. New York, Sunderland, MA Oxford University Press; Sinauer Associates; 2018. xviii, 334 pages p.

36. Wang HY, Chien MH, Huang HP, Chang HC, Wu CC, Chen PJ, et al. Distinct hepatitis $B$ virus dynamics in the immunotolerant and early immunoclearance phases. J Virol. 2010;84(7):3454-63.

37. Xiang D, Shen X, Pu Z, Irwin DM, Liao M, Shen Y. Convergent evolution of human-isolated H7N9 avian influenza a viruses. J Infect Dis. 2018;217(11): 1699-707.

38. Clavel F, Hance AJ. HIV drug resistance. N Engl J Med. 2004;350(10):1023-35.

39. Locarnini S, Zoulim F. Molecular genetics of HBV infection. Antivir Ther. 2010;15(Suppl 3):3-14.

40. Wen $\mathrm{H}$, Wang $\mathrm{HY}, \mathrm{He} \mathrm{X}$, Wu $\mathrm{Cl}$. On the low reproducibility of cancer studies. Natl Sci Rev. 2018;5(5):619-24.

41. Muth D, Corman VM, Roth H, Binger T, Dijkman R, Gottula LT, et al. Attenuation of replication by a 29 nucleotide deletion in SARS-coronavirus acquired during the early stages of human-to-human transmission. Sci Rep. 2018;8(1):15177.

42. Sung SC, Chao CY, Jeng KS, Yang JY, Lai MMC. The 8ab protein of SARS-CoV is a luminal ER membrane-associated protein and induces the activation of ATF6. Virology. 2009;387(2):402-13.

43. Wong HH, Fung TS, Fang S, Huang M, Le MT, Liu DX. Accessory proteins 8b and $8 a b$ of severe acute respiratory syndrome coronavirus suppress the interferon signaling pathway by mediating ubiquitin-dependent rapid degradation of interferon regulatory factor 3. Virology. 2018:515:165-75.

44. Le TM, Wong HH, Tay FP, Fang S, Keng CT, Tan YJ, et al. Expression, posttranslational modification and biochemical characterization of proteins encoded by subgenomic mRNA8 of the severe acute respiratory syndrome coronavirus. FEBS J. 2007:274(16):4211-22.

45. Chen C-Y, Ping Y-H, Lee H-C, Chen K-H, Lee Y-M, Chan Y-J, et al. Open Reading frame $8 \mathrm{a}$ of the human severe acute respiratory syndrome coronavirus not only promotes viral replication but also induces apoptosis. J Infect Dis. 2007;196(3):405-15.

46. Ceraolo C, Giorgi FM. Genomic variance of the 2019-nCoV coronavirus. J Med Virol. 2020;92(5):522-8.

47. Siu KL, Yuen KS, Castano-Rodriguez C, Ye ZW, Yeung ML, Fung SY, et al. Severe acute respiratory syndrome coronavirus ORF3a protein activates the NLRP3 inflammasome by promoting TRAF3-dependent ubiquitination of ASC. FASEB J. 2019;33(8):8865-77

48. Yue Y, Nabar NR, Shi CS, Kamenyeva O, Xiao X, Hwang IY, et al. SARScoronavirus open Reading frame-3a drives multimodal necrotic cell death. Cell Death Dis. 2018:9(9):904

49. Castano-Rodriguez C, Honrubia JM, Gutierrez-Alvarez J, DeDiego ML, NietoTorres $J \mathrm{~L}$, Jimenez-Guardeno JM, et al. Role of Severe Acute Respiratory Syndrome Coronavirus Viroporins E, 3a, and 8a in Replication and Pathogenesis. mBio. 2018;9(3).

\section{Publisher's Note}

Springer Nature remains neutral with regard to jurisdictional claims in published maps and institutional affiliations.
Ready to submit your research? Choose BMC and benefit from:

- fast, convenient online submission

- thorough peer review by experienced researchers in your field

- rapid publication on acceptance

- support for research data, including large and complex data types

- gold Open Access which fosters wider collaboration and increased citations

- maximum visibility for your research: over $100 \mathrm{M}$ website views per year

At $\mathrm{BMC}$, research is always in progress.

Learn more biomedcentral.com/submissions 